\title{
Mechanical properties of composites as functions of the syringe storage temperature and energy dose
}

Fernanda Oliveira CHAVES, Natália Coelho de FARIAS, Luciano Marcelo de Mello MEDEIROS, Roberta Caroline Bruschi ALONSO, Vinicius DI HIPÓLITO, Paulo Henrique Perlatti D'ALPINO

Biomaterials Research Group, Universidade Anhanguera de São Paulo, São Paulo, SP, Brazil.

Corresponding address: Paulo Henrique Perlatti D’Alpino - Universidade Anhanguera de São Paulo - Mestrado Profissional em Biomateriais em Odontologia

- Rua Maria Cândida, 1.813 - 6ª andar - Bloco G - São Paulo - SP - 02071-013 - Phone: +55 1129679058 - e-mail: paulodalpino@yahoo.com

Submitted: November 22, 2013 - Modification: May 29, 2014 - Accepted: June 7, 2014

\section{ABSTRACT}

$\mathrm{O}$ bjective: To investigate the mechanical properties of different classifications of composites indicated for posterior application as functions of the storage condition and of the energy dose. Material and Methods: Specimens $(8 \times 2 \times 2 \mathrm{~mm})$ were obtained according to the factors: I) Composites (3M ESPE): Filtek P60, Filtek Z350XT, and Filtek Silorane; II) Syringe storage conditions: room temperature, aged, oven, refrigerator, and freezer; and III) Energy dose: $24 \mathrm{~J} / \mathrm{cm}^{2}$ and $48 \mathrm{~J} / \mathrm{cm}^{2}$. After photoactivation, the specimens were stored at $37^{\circ} \mathrm{C}$ for $24 \mathrm{~h}$. After storage, a three-point bending test was carried out in a universal testing machine at $0.5 \mathrm{~mm} / \mathrm{min}$. Flexural strength $(\mathrm{S})$ and flexural modulus (E) were calculated. Data were analyzed by three-way ANOVA and Tukey's test $(\alpha=0.05)$. Results: Different storage conditions significantly affected the silorane composite for S; conversely, no effects were noted in terms of $\mathrm{E}$. The accelerated aging protocol significantly increased the S of Filtek P60 and Filtek Silorane, whereas storage in the oven significantly decreased the S for all of the composites tested. Filtek P60 was the only composite not affected by the lower storage temperatures tested for $S$, whereas for the silorane this parameter was impacted at the same conditions. The factor "dose" was not statistically significant. Conclusions: The syringe storage at different temperature conditions proved to influence mostly the flexural strength, a clinically important characteristic considering the posterior indication of the materials tested. The silorane composite should not be stored at lower temperatures.

Keywords: Composite resins. Physical properties. Storage of substances, products and materials. Aging.

\section{INTRODUCTION}

Advances in the mechanical properties of resin composites, associated with improvement in adhesive systems, have favored the clinical application of these restorative materials, especially in posterior teeth. In spite of these improvements, the mechanical properties of these materials vary considerably because of the differences in the composition of the organic matrix as well as the type, size, and packing of the inorganic fillers ${ }^{12}$. The introduction of new or modified dental products on the market requires the assurance that these materials can be stored for an extended period without any decrease in their performance that may affect safety and efficacy ${ }^{14}$. Shelf life is defined as the term or period during which a commodity remains suitable for the intended use ${ }^{9}$. On the other hand, expiration date is the termination of shelf life, after which a percentage of the product, i.e., medical devices, may no longer function as originally intended. In order to determine whether a certain product requires a shelf life and assign an expiration date, there are a number of different parameters that must be considered ${ }^{13}$. In this way, this product must be analyzed to determine if it is susceptible to degradation that would lead to functional failure and to determine the level of risk 
that the failure would present ${ }^{9}$.

Accelerated aging protocols are generally applied to new products to provide experimental data in order to estimate their clinical performance and shelf life claims. In these protocols, products are subjected to stresses that are more severe or more frequently applied than normal environmental or operational stresses for a shorter time ${ }^{14}$. Studies on accelerated aging combined with basic stability information on the components (functional chemical groups organized in diverse ways crystalline or amorphous - along with additives such as antioxidants, inorganic fillers, plasticizers, colorants, and processing aids ${ }^{14}$ ) may be used to support tentative expiration dates ${ }^{5}$. These variations, combined with the different protocols of clinical applications and oral environment, determine the degradation process ${ }^{14}$. In a previous study ${ }^{6}$, it was pointed out that the material condition may affect the propagation stage (second stage of the polymerization reaction attributed to the propagating radical), in which the free radicals react successively with monomers to form a threedimensional network.

Clinicians tend to store the restorative materials at low temperatures, especially the resin composites, in order to "prolong" their shelf life ${ }^{15}$. However, these cooled restorative materials are used immediately after being removed from the refrigerator ${ }^{10}$. In addition, their manipulative properties are modified, considering that some of the composites are too sticky and tend to flow less at lower temperatures. A consensus that the rheological properties of resin-composites play an important role in terms of restoration longevity exists ${ }^{17}$. The viscosity of a resin-composite is directly related to its handling characteristics (placement and shaping on the restored site $)^{19}$. In spite of the composite stickiness, manufacturers have modified the composite viscosity, as this manipulative characteristic may allow the formation of macroscopic spaces and microscopic porosities in the restorations ${ }^{23}$. This can be partially attributed to the "pull-back" effect ${ }^{1}$. Clinically, an unpolymerized resin-composite is applied and exposed while in a varied viscous state and variations in the conversion values can be reached as the rheological properties of composites influence the radical mobility, depending on the viscosity ${ }^{6}$. In this way, no objective criteria to support the indication of high viscous composites or "compactable" composites have been provided ${ }^{16}$. In addition, the influence of the energy dose as a function of the material condition needs further investigation. As the kinetics of polymerization is dependent upon the energy dose, it could be expected that the longer light exposure time would somehow allow a higher end conversion?, irrespective of the material condition.

This study evaluated the mechanical properties (flexural strength and flexural modulus) of different classifications of composites indicated for posterior application as functions of the storage condition

\begin{tabular}{|c|c|c|c|c|c|c|}
\hline Composite & Classification & Organic matrix & Fillers & Filler type & $\begin{array}{c}\begin{array}{c}\% \text { fillers } / w t \\
\text { (vol..) }^{*}\end{array}\end{array}$ & $\begin{array}{l}\text { Lot \# } \\
\text { (expiration } \\
\text { date) }\end{array}$ \\
\hline \multirow[t]{2}{*}{$\begin{array}{l}\text { Filtek P60 } \\
\text { (P60) }\end{array}$} & Hybrid & $\begin{array}{c}\text { BisGMA, UDMA, } \\
\text { BisEMA }\end{array}$ & $0.01-3.5 \mu \mathrm{m}$ & Silica / & 83.0 & N126976 \\
\hline & & & $\begin{array}{c}\text { (average } 0.6 \\
\mu \mathrm{m})\end{array}$ & Zirconia & $(61.7)$ & Sep./2012 \\
\hline \multirow[t]{3}{*}{$\begin{array}{l}\text { Filtek Z350XT } \\
\qquad(\mathrm{XT})\end{array}$} & Nanofilled & $\begin{array}{c}\text { BisGMA, TEGDMA, } \\
\text { UDMA, BisEMA(6), } \\
\text { PEGDMA }\end{array}$ & Silica: 20 nm & Zirconia / Silica & 72.5 & 9YE \\
\hline & & & Zirconia: & $\begin{array}{c}\text { Aggregated clusters } \\
\text { and nano-fillers }\end{array}$ & $(55.6)$ & Feb./2012 \\
\hline & & & $4-11 \mathrm{~nm}$ & & & \\
\hline \multirow[t]{2}{*}{$\begin{array}{c}\text { Filtek Silorane } \\
\text { (FS) }\end{array}$} & Microhybrid & Silorane ${ }^{* *}$ & 0.47 (average) & Quartz, & 76.0 & N128528 \\
\hline & & & & Yttrium fluoride & $(58.0)$ & Jul./2011 \\
\hline
\end{tabular}

BisGMA: bisphenol-glycidyl-methacrylate; UDMA: urethane-dimethacrylate; BisEMA: bisphenol-a-ethoxy dimethacrylate; TEGDMA: triethyleneglycol dimethacrylate; PEGDMA: Poly(ethylene glycol) dimethacrylate

*Manufacturer's information (3M ESPE, St. Paul, MN, USA)

**Bis-3,4-EpoxycyclohexylethylPhenyl-Methylsilane and 3,4-Epoxycyclohexylcyclopolymethylsiloxane Note: The brand name Filtek Silorane is used in other countries as Filtek LS and Filtek P90

Figure 1- Description of the composites used in this study 
(room temperature, aged, oven, refrigerator, and freezer) and the energy dose. The following research hypotheses were tested: (1) the storage temperature can influence the flexural strength of the composites tested; (2) the flexural modulus of the composites stored at different temperatures will be inferior to that observed at room temperature; (3) the energy dose influences the mechanical properties of the composites, irrespective of the storage temperature.

\section{MATERIAL AND METHODS}

\section{Experimental design}

In this in vitro study, mechanical characterizations were performed according to the factors: (1) composites, at three levels: Filtek P60 (P60), Filtek Z350XT (XT), and Filtek Silorane (FS); and (2) material conditions, at five levels: I - control (room temperature - RT, $22^{\circ} \mathrm{C}$ ), II - accelerated aged composites (AA), III - oven at $37^{\circ} \mathrm{C}$ for $24 \mathrm{~h}(\mathrm{OV})$, IV - refrigerator at $2^{\circ} \mathrm{C}$ for $24 \mathrm{~h}(\mathrm{RF})$, and $\mathrm{V}$ - freezer at $-15^{\circ} \mathrm{C}$ for $24 \mathrm{~h}(\mathrm{FZ})$; (3) energy dose, at two levels: I $-24 \mathrm{~J} / \mathrm{cm}^{2}$, II $-48 \mathrm{~J} / \mathrm{cm}^{2}$. The characteristics of the resin composites selected are described in the Figure 1 . The schematic representation of the experimental design is depicted in Figure 2. Thirty experimental groups were obtained of the product among the combinations of the factors under study.

\section{Accelerated aging protocol}

The composites underwent a simplified protocol for accelerated aging (also called the "10-degree rule") according to the collision theory based on the Arrhenius model. The composite syringes were stored at $37^{\circ} \mathrm{C}$ in an oven for 12 weeks. According to Clark $^{5}$ (1991), the accelerated aging protocol can be calculated by means of a mathematical formula, as follows:

$$
\begin{aligned}
& r=Q 10((R T-E T) / 10) \\
& \text { where, } \\
& r=\text { accelerated aging rate; } \\
& R T=\text { room temperature }\left(22^{\circ} \mathrm{C}\right) ; \\
& E T=\text { elevated temperature }\left(37^{\circ} \mathrm{C}\right) ; \\
& Q_{10}=\text { reaction rate coefficient }(2) .
\end{aligned}
$$

According to this protocol, for each $10^{\circ} \mathrm{C}$ increase above the room temperature, the reaction rate doubles $^{5}$. In this way, as the $R T$ was equivalent to $22^{\circ} \mathrm{C}, r$ corresponds to $2^{1.5}(37-22=15 ; 15 / 10=1.5)$. Thus, the accelerated aging rate was based on the increase of $15^{\circ} \mathrm{C}$ in the storage temperature, which is 1.5 squared $\left(r=1.5^{2}=2.83\right)$. Finally, in order to estimate the aging time equivalent to that which would occur at room temperature, $r$ was multiplied

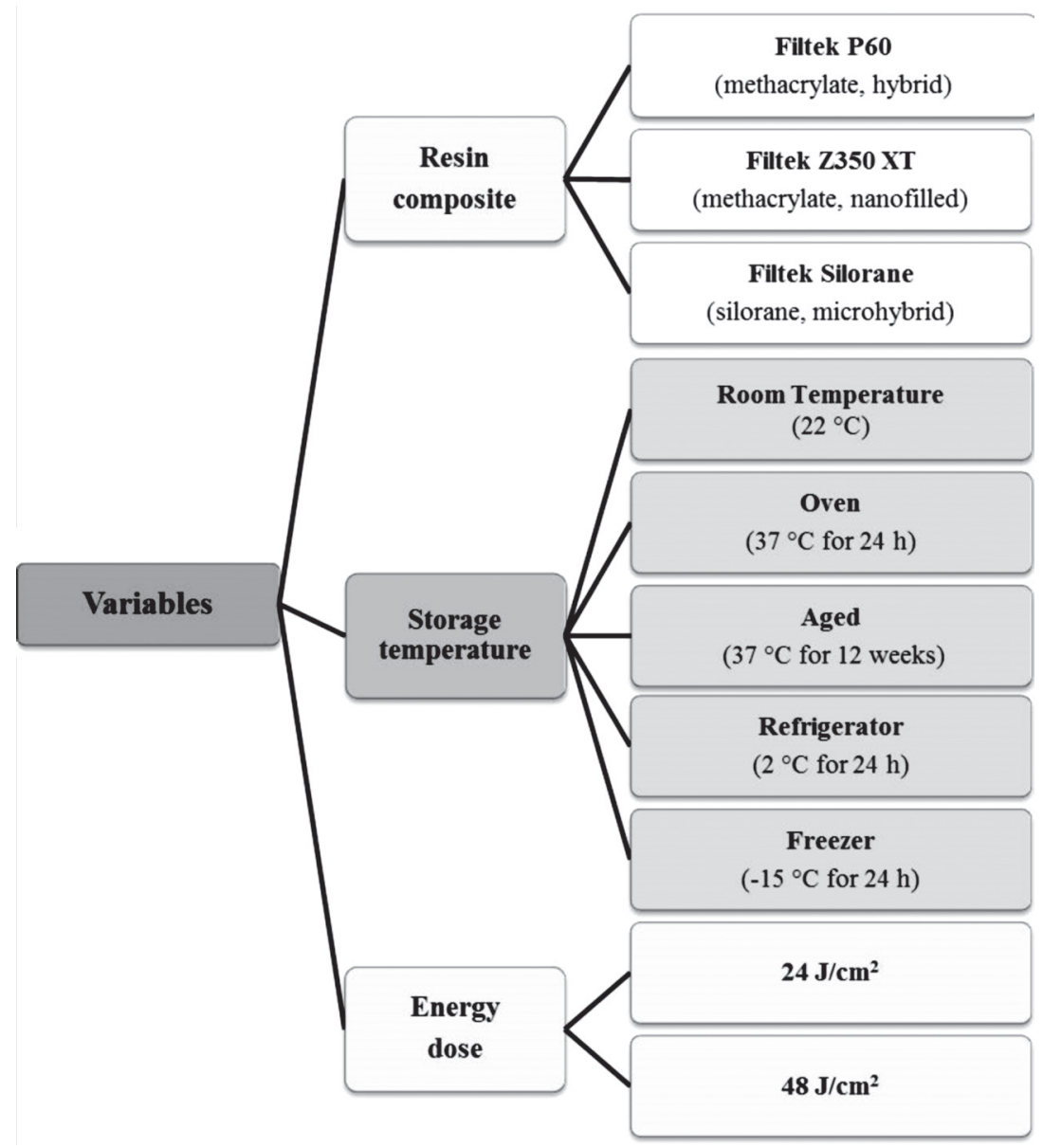

Figure 2- Schematic representation of the experimental design 
by the storage time $(12 \times 2.83)$, which represents approximately 9 months of aging. It is important to mention that the same lot numbers were used for the material condition levels of "room temperature" and "aged". In addition, care was taken to ensure that the composites were not expired after the accelerated aging protocol (simulating a 9-month shelf life) $)^{7}$.

\section{Specimen fabrication}

After the storage time respective to the experimental groups had elapsed, the syringes were kept untouched until room temperature was reached. An infrared thermometer (General Tools \& Instruments, IRT-206 Gun-style Infrared Thermometer, New York, NY, USA) was used to evaluate the syringe temperatures. Flexural strength evaluations differed slightly from that described in ISO 4049. The composites were applied to a Teflon mold $(8 \times 2 \times 2 \mathrm{~mm})$, positioned over a polyester strip $(n=10)$. After filling the mold to excess, the material surface was covered with a Mylar strip and a glass slide and compressed to extrude excess material. All restorative materials were photoactivated using an LED light-curing unit (Bluephase, Ivoclar Vivadent, Schaan, Liechtenstein) with a power density of 1200 $\mathrm{mW} / \mathrm{cm}^{2}$ for $20 \mathrm{~s}$ (energy dose of $24 \mathrm{~J} / \mathrm{cm}^{2}$ ) or for $40 \mathrm{~s}$ (energy dose of $48 \mathrm{~J} / \mathrm{cm}^{2}$ ). Prior to testing and throughout the experiment, the power density was monitored using a handheld radiometer (Model 100, Demetron Research Corp., Danbury, CT, USA). All of the composites selected were shade A3 (an exception was XT: shade A3B). Specimens were then stored in distilled water at $37^{\circ} \mathrm{C}$ for $24 \mathrm{~h}$.

\section{Flexural strength test (S)}

Prior to the test, the specimen dimensions were measured using a digital caliper (Digimatic Caliper $C D$, Mitutoyo, Japan). The three-point bending test was carried out in a universal testing machine (Instron model 3342, Instron Corp., Canton, MA, USA) at $0.5 \mathrm{~mm} / \mathrm{min}$ and $5 \mathrm{~mm}$ span between supports. S (s) was calculated as follows:

$$
\sigma=\frac{3 F \times L}{2 b \times h^{2}}
$$

Flexural Modulus $(E)$ was calculated by:

$$
E=\frac{L^{3}}{4 b \times h^{3}} \times \frac{F}{Y}
$$

where $F$ is the maximum strength in $\mathrm{N}$; $L$ the distance between the rests; $b$ the width of the specimen; $h$ the height of the specimen; and $F / Y$ the slope of the linear part of the stress-strain curve. Statistical analysis of $S$ and $E$ was performed with three-way ANOVA and Tukey post-hoc test $(\alpha=5 \%)$.

\section{RESULTS}

Figure 3 shows data of $S$ regarding the three factors: composite $x$ syringe storage condition $x$ energy dose. The results of $S$ are listed in Table 1. The highest $S$ mean was noted for P60 AA (329.2 MPa), and the lowest for Z250 OV $(117.3 \mathrm{MPa})$. The interaction between the factors "composites" and "material condition" was significant. However, the factor "dose" and the triple interaction among the factors were not significant. All of the composites exhibited statistically

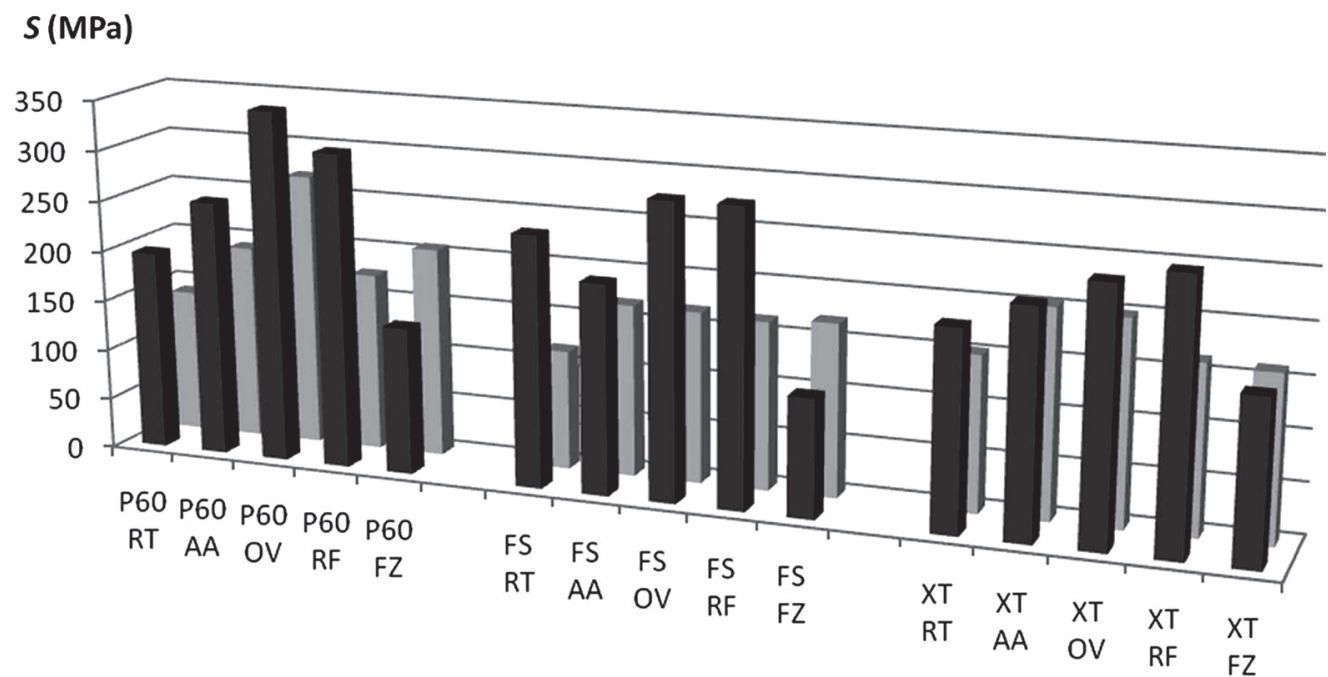

$24 \mathrm{~J} / \mathrm{cm}^{2}-48 \mathrm{~J} / \mathrm{cm}^{2}$

Figure 3- Graphic of flexural strength (MPa) considering all factors: I. Composites: Filtek P60 (P60), Filtek Silorane (FS) and Filtek Z350XT (XT); II. Syringe storage conditions: Room Temperature (RT), Accelerated aging (AA), Oven 24 h (OV), Refrigerator $24 \mathrm{~h}(\mathrm{RF})$, Freezer $24 \mathrm{~h}$ (FZ); III. Energy doses: $24 \mathrm{~J} / \mathrm{cm}^{2}$ and $48 \mathrm{~J} / \mathrm{cm}^{2}$ 
equivalent $S$ means at room temperature (RT) and freezer $(F Z)$ storage $(p>0.05)$. The $S$ means of $\mathrm{P} 60$ were not influenced by the storage at lower temperatures (RF and FZ), whereas for the silorane composite $S$ mean significantly decreased $(p<0.05)$. On the other hand, only the storage at FZ significantly decreased $S$ means of XT; the $S$ mean was not influenced by the RF storage in comparison to that at RT ( $p>0.05)$. All the composites exhibited significantly decreased in the $S$ means when the syringes were stored in the oven at $37^{\circ} \mathrm{C}$ for $24 \mathrm{~h}$ compared to that of at RT $(p<0.05)$. On the other hand, the simplified protocol of accelerated aging led to a significant increase in the $S$ means for P60 and for Filtek Silorane $(p<0.05)$.

Figure 4 shows the data of $E$ regarding the three

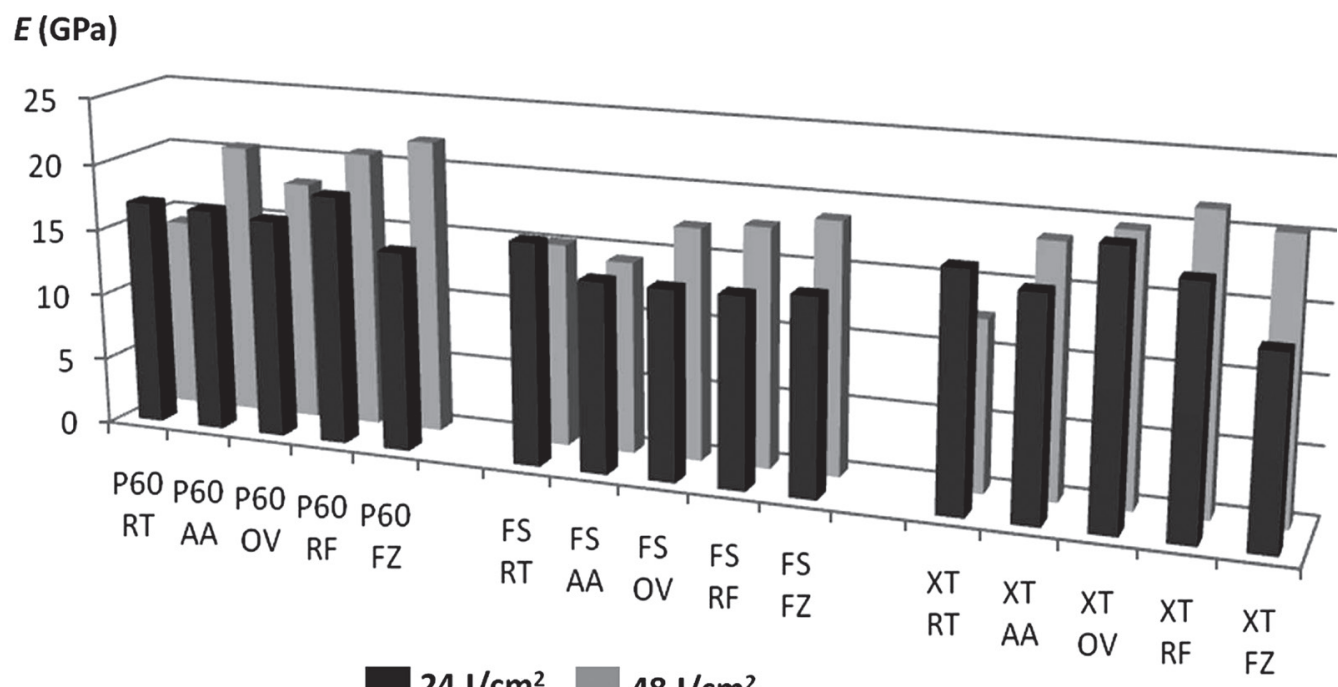

$24 \mathrm{~J} / \mathrm{cm}^{2} \quad 48 \mathrm{~J} / \mathrm{cm}^{2}$

Figure 4- Graphic of flexural modulus (GPa) considering all factors: I. Composites: Filtek P60 (P60), Filtek Silorane (FS) and Filtek Z350XT (XT); II. Syringe storage conditions: Room Temperature (RT), Accelerated aging (AA), Oven 24 h (OV), Refrigerator $24 \mathrm{~h}(\mathrm{RF})$, Freezer $24 \mathrm{~h}$ (FZ); III. Energy doses: $24 \mathrm{~J} / \mathrm{cm}^{2}$ and $48 \mathrm{~J} / \mathrm{cm}^{2}$

Table 1- Mean of flexural strength (S) regarding interaction Composite x Syringe storage condition

\begin{tabular}{clll}
\hline $\begin{array}{c}\text { Composites I } \\
\text { Storage condition }\end{array}$ & Filtek P60 & Filtek Silorane & Filtek Z350XT \\
\hline Room Temperature (RT) & $224.9(41.2)^{\mathrm{bA}}$ & $227.1(42.7)^{\mathrm{bA}}$ & $208.8(27.7)^{\mathrm{abA}}$ \\
Accelerated aging (AA) & $329.2(43.0)^{\mathrm{aA}}$ & $290.6(59.7)^{\mathrm{aAB}}$ & $255.3(52.4)^{\mathrm{aB}}$ \\
Oven 24 h (OV) & $143.2(24.6)^{\mathrm{cA}}$ & $117.3(24.1)^{\mathrm{dB}}$ & $156.1(21.4)^{\mathrm{dA}}$ \\
Refrigerator 24 h (RF) & $231.2(51.0)^{\mathrm{bA}}$ & $169.1(27.4)^{\mathrm{cB}}$ & $202.1(37.5)^{\mathrm{bcA}}$ \\
Freezer 24 h (FZ) & $191.7(35.7)^{\mathrm{bA}}$ & $167.9(15.7)^{\mathrm{cA}}$ & $163.4(30.7)^{\mathrm{cdA}}$ \\
\hline
\end{tabular}

Mean values followed by different capital letters in row and small letters in column: significant $(p<0.05)$

( ): standard deviation

Table 2- Mean of flexural modulus (E) regarding interaction Composite $x$ Syringe storage condition

\begin{tabular}{cccc}
\hline Composites I & Filtek P60 & Filtek Silorane & Filtek Z350XT \\
Storage condition & & & $16.9(1.2)^{\mathrm{bA}}$ \\
\hline Room Temperature (RT) & $16.9(2.5)^{\mathrm{bcA}}$ & $15.4(2.3)^{\mathrm{abA}}$ & $19.0(3.6)^{\mathrm{abA}}$ \\
Accelerated aging (AA) & $17.6(2.2)^{\mathrm{bcA}}$ & $14.2(1.5)^{\mathrm{bB}}$ & $13.3(2.4)^{\mathrm{cA}}$ \\
Oven 24 h (OV) & $14.6(4.1)^{\mathrm{cA}}$ & $14.9(4.2)^{\mathrm{bA}}$ & $19.2(1.4)^{\mathrm{abA}}$ \\
Refrigerator 24 h (RF) & $19.3(1.9)^{\mathrm{abA}}$ & $15.9(1.9)^{\mathrm{abB}}$ & $21.0(1.9)^{\mathrm{aA}}$ \\
\hline Freezer 24 h (FZ) & $21.5(1.2)^{\mathrm{aA}}$ & $18.3(1.7)^{\mathrm{aB}}$ & 2 \\
\hline
\end{tabular}

Mean values followed by different capital letters in row and small letters in column: significant $(p<0.05)$

( ): standard deviation 
factors: composite $x$ syringe storage condition $x$ energy dose. The highest $E$ mean was noted for P60 FZ (21.5 GPa) and the lowest for XT OV (13.3 GPa). The results of $E$ are listed in Table 2 . The interaction between the factors "composites" and "material condition" was significant. The factor "dose" and the triple interaction among the factors were not significant. The factor "storage condition" produced no effect in the $E$ means of the silorane composite $(p>0.05)$. For P60 and XT, storage at OV significantly decreased the $E$ means, whereas the storage at FZ significantly increased the flexural modulus $(p<0.05)$.

\section{DISCUSSION}

Although the composites tested presented equivalent $S$ means at room temperature, the results were material-dependent when considering the different storage conditions. Filtek Silorane was the composite most influenced by the storage condition. The simplified protocol of accelerated aging produced an increase in $S$ for the silorane composite whereas all of the other conditions (oven, refrigerator, and freezer for $24 \mathrm{~h}$ ) produced a significant reduction in this mechanical parameter. On the other hand, Filtek P60 was not influenced by neither of the two lower temperatures whereas Filtek Z350XT showed a significant reduction in $S$ only when stored at the freezer at $37^{\circ} \mathrm{C}$ for $24 \mathrm{~h}$. Aging the Filtek $\mathrm{P} 60$ produced a significant increase in $S$. The composite Filtek Z350XT was the only composite not influenced by the simplified protocol of accelerated aging. Storing all of the composites in the oven produced a significant reduction in $S$. Thus, the first research hypothesis raised in the present study was upheld by the experimental data as the storage temperature influences the flexural strength of the composites tested.

The simplified approach for accelerated aging used in the present study was based on conducting testing at a single accelerated temperature and then employing the rule stating that the rate of a chemical reaction will increase by a factor $\mathrm{Q}_{10}$ (usually of 2 ), which represents a doubling of the reaction rate for every $10^{\circ} \mathrm{C}$ increase in temperature ${ }^{14}$. This type of conservative relationship is indicated for a variety of medical polymers that have been previously characterized ${ }^{14}$. The degradation may be primarily chemical - a combination of effects arising from oxidative chain scission, oxidation hydrolysis, changes in crystallinity, and other factors that may be environmentally dependent ${ }^{11}$. Chemical crosslinking links molecules, while crystallinity and fillers introduce physical limitations to monomer mobility ${ }^{4}$. Under these variations, the rate of radical propagation eventually becomes diffusionlimited and the polymerization rate decelerates, often providing only a limited conversion even in the presence of an unreacted monomer and a population of radicals ${ }^{27}$. In this way, the crosslinking will be restricted by the molecular response, depending on the storage temperature, since the degree of freedom for monomer motions is reduced as the these monomers become irreversibly linked. This was particularly true in the accelerated aging composites as significant variations in the flexural strength occurred in comparison to that at room temperature (Table 1 ).

In contrast to those results noted after the accelerated aging protocol, in which the $S$ means increased, the $S$ means obtained after storage in the oven at $37^{\circ} \mathrm{C}$ for $24 \mathrm{~h}$ significantly decreased for all of the tested composites. One would argue that an enhanced flexural strength would be obtained after the storage in the oven at $37^{\circ} \mathrm{C}$ as the result of an increased conversion given by temperaturederived mobility of polymeric chains and consequent entrapped radical engagement ${ }^{2}$. Therefore, it is reasonable to infer that the composites underwent a chemical degradation at shorter storage time ( $24 \mathrm{~h}$ ) before a change in the crystallinity would occur throughout the 12-week storage at the same temperature (aging protocol). It could be argued that, by significantly increasing the flexural strength after aging the composite syringes of Filtek P60 and Filtek Silorane would provide better clinical performance. Conversely, the increased flexural strength may not represent improved mechanical properties, despite the equivalence in terms of flexural modulus when compared to that of the means at room temperature. In this way, it was clear that these contradictory results when comparing shorter and longer storages in the oven at $37^{\circ} \mathrm{C}$ have in common the fact that both storage conditions interfere in the composite stability.

Many aspects must be considered when analyzing the results. It is important to highlight that the mechanical properties of composites tested depend on the properties of their constituent materials ${ }^{25}$. Thus, when comparing the different composites, the large ranges in the $S$ means were due to the different classifications in the composite formulations, although all tested materials are indicated for restoring posterior teeth. Filtek Silorane is filled with a combination of fine quartz particles and radiopaque yttrium fluoride, being classified as a microhybrid resin composite. The concentration of filler particles in this composite is $76.0 \%$ by weight. The filler in Filtek P60 is zirconia/ silica in a concentration of $83.0 \%$ by weight, classified as a hybrid composite. Filtek Z350XT is a nanofilled composite with $72.5 \%$ of fillers. As these materials are essentially polymeric in nature, their laboratorial and clinical performances are related to the rate of degradation of their inherent 
structure and configuration over time. In contrast to methacrylate-base composites, the siloranebased composite contains cationic ring-opening monomers, which represent a compensating mechanism for shrinkage stress achieved during polymerization ${ }^{30}$. This monomer system was obtained from the reaction of oxirane and siloxane molecules $^{30}$. This resin composite combines two main advantages: low polymerization shrinkage, due to the ring-opening oxirane, and an increased hydrophobicity due to the siloxane compound. The silorane-based composite revealed a decreased water sorption, solubility, and associated diffusion coefficient compared to those observed when methacrylate-based composites were tested ${ }^{24}$.

The elastic modulus of low-shrinking composites and methacrylate-based composite was recently reported as being similar, despite differences in the formulation, even though the silorane composite has higher molecular weight and higher filler content $^{29}$. In another study, it was also revealed that the silorane material presents relatively higher flexural strength/modulus and fracture toughness but relatively lower compressive strength and hardness than some methacrylate-based restorative materials ${ }^{20}$. In the present study, no significance was noted among the composites in terms of $E$ at room temperature. In addition, none of the composites had the means of flexural modulus affected by the accelerated aging protocol. Different from that observed for flexural strength, all of the storage conditions produced no effect on $E$ of the silorane composite. On the other hand, Filtek P60 and Filtek Z350XT were affected by storage in the oven at $37^{\circ} \mathrm{C}$ for $24 \mathrm{~h}$, significantly decreasing $E$, whereas the storage in the freezer significantly increased $E(p<0.05)$. In this way, the second research hypothesis, that the flexural modulus of the composites stored at different temperatures will be inferior to that observed at room temperature, was also rejected.

In spite of the similarity in terms of the flexural modulus, the flexural strength of the Filtek Silorane was the only composite negatively influenced by both the lower temperatures and storage conditions. The reasons that explain the lower flexural strength means rely on the fact that the silorane monomer presents a polymerization reaction with a slow onset $^{28}$. In addition, the lower temperatures may impact the initial activation energy necessary to form the activated complex, which represents the first two stages of the composite polymerization initiation and activation ${ }^{3}$. This somehow leads to a more reduced extent of the composite monomer conversion that may impact the quality of the polymerization process ${ }^{26}$. Resin conversion should be usually optimized in order to resist deterioration of mechanical and chemical properties; strength, hardness, stiffness, and wear resistance. Even the exposure of a $48 \mathrm{~J} / \mathrm{cm}^{2}$ energy dose was not sufficient to compensate the impact on the polymerization process. In this way, based on the results of the present study, it is not recommended to store Filtek Silorane at low temperatures.

It has been advocated that refrigerated syringe composites should be warmed to at least room temperature before clinical application as this procedure might disrupt the material characteristics ${ }^{15}$. It is important to point out that the composite syringes underwent the temperature treatment and not the polymerized specimens. In addition, the specimens were produced only when the syringes were at room temperature. Although dental manufacturers recommend that composites be stored at room temperature ${ }^{10}$, all of the variables that include storage temperature and transportation conditions are not under control. The effect of these variables over the composite clinical performance can be minimized if properly considered. These variables can have an effect in a way that may adversely affect the composite safety or performance ${ }^{18}$. Temperature variations, relative humidity, ventilation, visible light and other radiation, vibration, shock, temperature, and humidity are other important factors to be considered, especially when a dental product is shipped between various climatic zones ${ }^{22}$. According to Clark $^{5}$ (1991), depending on the shipping or storage conditions, a breakage in a certain medical device, a failure of the barrier properties of a sterile package or degeneration of the device itself may occur. In this way, the question as to how new the dental products available to the clinicians are remains unanswered.

The third research hypothesis, which anticipated that the energy dose influences in the mechanical properties of the composites, irrespective of the storage temperature, was rejected. According to the statistical analysis, the factor "dose" and the triple interaction among the factors were not significant for both the parameters tested. A reasonable explanation was that, in the present study, a $24 \mathrm{~J} /$ $\mathrm{cm}^{2}$ energy dose was sufficient to guarantee the composite polymerization of 2-mm thick specimens. Clinically, it would be important to obtain well polymerized composites with enhanced mechanical properties. On the other hand, an energy dose of $48 \mathrm{~J} / \mathrm{cm}^{2}$ should be used with caution by clinicians considering the increase in the polymerization stress generated when higher energy doses are applied. In addition, the specimens exposed to a low energy dose were stored at $37^{\circ} \mathrm{C}$ for $24 \mathrm{~h}$ after photoactivation, and this fact may allow an increased monomer conversion and enhanced mechanical properties similar to that of obtained when a higher energy dose was applied ${ }^{21}$. In a 
previous study ${ }^{7}$, it was pointed out that the energy dose associated with the bonding approach are determinant factors in terms of the presence of internal gaps for both the methacrylate-based composite and for the silorane-based one.

The present study deals with a clinical relevant issue as the syringe storage conditions tested influenced the mechanical properties of different classifications of resin composites. Storage as a function of the temperature proved to influence mostly the composite flexural strength, possibly due to changes in the chemical stability and/or crystallinity (especially at higher temperatures) of the composites and also due to the impact of the quality of the polymerization process (lower temperatures). Although the dental manufacturers may not be able to control all of the variables like the storage temperature and the transportation conditions, their effect on the composite clinical performance can be minimized if properly considered by the clinicians ${ }^{8}$. Extrapolations to clinically support and validate the results using different resincomposite categories and filler contents need to be done with caution since future studies are required to evaluate the performance of these composites.

\section{CONCLUSION}

Within the limitations of this study, the following can be concluded:

The storage condition influences the flexural strength and the flexural modulus of the composites in comparison to that observed at room temperature.

The energy dose does not influence the mechanical properties, irrespective of the storage condition.

The aging protocol improves the flexural strength of Filtek P60 and Filtek Silorane, whereas storage in the oven at $37^{\circ} \mathrm{C}$ for $24 \mathrm{~h}$ impacts the same parameter for all of the composites tested.

It seems that the silorane composite should not be stored at lower temperatures, whereas the Filtek P60 is not impacted by these temperatures.

\section{REFERENCES}

1- Al-Sharaa KA, Watts DC. Stickiness prior to setting of some light cured resin-composites. Dent Mater. 2003;19(3):182-7.

2- Bagis $\mathrm{YH}$, Rueggeberg FA. The effect of post-cure heating on residual, unreacted monomer in a commercial resin composite. Dent Mater. 2000;16(4):244-7.

3- Bayne SC, Thompson JY. Biomaterials. Book. In: Roberson TM, Heymann HO ,Ritter AV. editors. Biomaterials. St. Louis: The CV Mosby Co.; 2006. p. 135-242.

4- Charton C, Falk V, Marchal P, Pla F, Colon P. Influence of Tg, viscosity and chemical structure of monomers on shrinkage stress in light-cured dimethacrylate-based dental resins. Dent Mater. 2007;23(11):1447-59.

5- Clark GS. Shelf life of medical devices, guidance document. Silver Spring: Division of Small Manufacturers, International and Costumer Assistance/FDA; 1991. 24 pages.

6- D'Alpino PH, Bechtold J, Santos PJ, Alonso RC, Di Hipólito V, Silikas N, et al. Methacrylate- and silorane-based composite restorations: hardness, depth of cure and interfacial gap formation as a function of the energy dose. Dent Mater. $2011 ; 27(11): 1162-9$. 7- D'Alpino PH, Vismara MV, Gonzalez AH, Graeff CF. Free radical entrapment and crystallinity of resin composites after accelerated aging as a function of the expiration date. J Mech Behav Biomed Mater. 2014;36:82-9.

8- D'Alpino PH, Vismara MV, Mello LM, Hipólito VD, González AH, Graeff CF. Resin composite characterizations following a simplified protocol of accelerated aging as a function of the expiration date. J Mech Behav Biomed Mater. 2014;35C:59-69.

9- Donohue J, Apostolou S. Shelf-life prediction for radiationsterilized plastic devices. Med Dev Diag Indu. 1990;12(1):124-9. 10- Faria-e-Silva AL, Piva E, Moraes RR. Time-dependent effect of refrigeration on viscosity and conversion kinetics of dental adhesive resins. Eur J Dent. 2010;4(2):150-5.

11- Ferracane JL. Hygroscopic and hydrolytic effects in dental polymer networks. Dent Mater. 2006;22(3):211-22.

12- Frauscher KE, Ilie N. Depth of cure and mechanical properties of nano-hybrid resin-based composites with novel and conventional matrix formulation. Clin Oral Investig. 2012;16:1425-34.

13- Gillen KT, Clough RL, Wise J. Extrapolating accelerated thermal aging results: a critical look at the arrhenius method. Polymer Prepr. 1993;34(2):185.

14- Hemmerich KJ. General aging theory and simplified protocol for accelerated aging of medical devices [online]. Medical Device and Diagnostic Industry. 1998 July 1. [cited 2013 Nov. 22]. Available from: http://www.mddionline.com/article/general-aging-theoryand-simplified-protocol-accelerated-aging-medical-devices.

15- Jafarzadeh-Kashi TS, Mirzaii M, Erfan M, Fazel A, Eskandarion $S$, Rakhshan V. Polymerization behavior and thermal characteristics of two new composites at five temperatures: refrigeration to preheating. J Adv Prosthodont. 2011;3(4):216-20.

16- Jordan RE, Suzuki M. The ideal composite material. J Can Dent Assoc. 1992;58(6):484,487.

17- Kaleem M, Satterthwaite JD, Watts DC. A method for assessing force/work parameters for stickiness of unset resin-composites. Dent Mater. 2011;27(8):805-10.

18- Kommanaboyina B, Rhodes CT. Trends in stability testing, with emphasis on stability during distribution and storage. Drug Dev Ind Pharm. 1999;25(7):857-68.

19- Lee JH, Um CM, Lee IB. Rheological properties of resin composites according to variations in monomer and filler composition. Dent Mater. 2006;22(6):515-26.

20- Lien W, Vandewalle KS. Physical properties of a new siloranebased restorative system. Dent Mater. 2010;26(4):337-44.

21- Lu H, Stansbury JW, Bowman CN. Towards the elucidation of shrinkage stress development and relaxation in dental composites. Dent Mater. 2004;20(10):979-86. 
22- Lucas TI, Bishara RH, Seevers RH. A stability program for the distribution of drug products. Pharm Tech. 2004;68-73. Available from: http://www.pharmtech.com/pharmtech/data/ articlestandard/pharmtech/282004/103355/article.pdf.

23- Opdam NJ, Roeters JJ, Peters TC, Burgersdijk RC, Kuijs RH. Consistency of resin composites for posterior use. Dent Mater. 1996;12(6):350-4.

24- Palin WM, Fleming GJ, Burke FJ, Marquis PM, Randall RC. The influence of short and medium-term water immersion on the hydrolytic stability of novel low-shrink dental composites. Dent Mater. 2005;21(9):852-63.

25- Peris AR, Mitsui FH, Amaral CM, Ambrosano GM, Pimenta LA. The effect of composite type on microhardness when using quartz-tungsten-halogen (qth) or led lights. Oper Dent. 2005;30(5):649-54.
26- Prasanna N, Pallavi Reddy Y, Kavitha S, Lakshmi Narayanan L. Degree of conversion and residual stress of preheated and roomtemperature composites. Indian J Dent Res. 2007;18(4):173-6. 27- Rueggeberg F. Contemporary issues in photocuring. Compend Contin Educ Dent. 1999;25:S4-15.

28- Stansbury JW, Trujillo-Lemon M, Lu H, Ding X, Lin Y, Ge J. Conversion-dependent shrinkage stress and strain in dental resins and composites. Dent Mater. 2005;21(1):56-67.

29- Tantbirojn D, Pfeifer CS, Braga RR, Versluis A. Do low-shrink composites reduce polymerization shrinkage effects? J Dent Res. 2011;90(5):596-601.

30- Weinmann W, Thalacker C, Guggenberger R. Siloranes in dental composites. Dent Mater. 2005;21(1):68-74. 